\title{
A New Approach for Unqualified Salted Sea Cucumber Identification: Integration of Image Texture and Machine Learning under the Pressure Contact
}

\author{
Huihui Wang $\mathbb{D}^{1,2,3,4}$ Xueyu Zhang $\mathbb{D}^{1,2,3,4}$ Pengpeng Li $\mathbb{D}^{1,2,3,4}$ Jialiang Sun $\mathbb{D}^{1,2,3,4}$ \\ Pengtao Yan $\mathbb{D}^{1,2,3,4}$ Xu Zhang $\mathbb{D}^{1,2,3,4}$ and Yanqiu Liu $\mathbb{D}^{1,2,3,4}$ \\ ${ }^{1}$ School of Mechanical Engineering \& Automation, Dalian Polytechnic University, Dalian 116034, China \\ ${ }^{2}$ School of Food Science and Technology, National Engineering Research Center of Seafood, Dalian Polytechnic University, \\ Dalian 116034, China \\ ${ }^{3}$ Collaborative Innovation Center of Seafood Deep Processing, Dalian 116034, China \\ ${ }^{4}$ Engineering Research Center of Seafood of Ministry of Education of China, Dalian 116034, China
}

Correspondence should be addressed to Yanqiu Liu; dlqiu@163.com

Received 27 August 2020; Revised 22 October 2020; Accepted 24 October 2020; Published 12 November 2020

Academic Editor: Eduard Llobet

Copyright (C) 2020 Huihui Wang et al. This is an open access article distributed under the Creative Commons Attribution License, which permits unrestricted use, distribution, and reproduction in any medium, provided the original work is properly cited.

At present, rapid, nondestructive, and objective identification of unqualified salted sea cucumbers with excessive salt content is extremely difficult. Artificial identification is the most common method, which is based on observing sea cucumber deformation during recovery after applying-removing pressure contact. This study is aimed at simulating the artificial identification method and establishing an identification model to distinguish whether the salted sea cucumber exceeds the standard by means of machine vision and machine learning technology. The system for identification of salted sea cucumbers was established, which was used for delivering the standard and uniform pressure forces and collecting the deformation images of salted sea cucumbers during the recovery after pressure removal. Image texture features of contour variation were extracted based on histograms (HIS) and gray level cooccurrence matrix (GLCM), which were used to establish the identification model by combining general regression neural networks (GRNN) and support vector machine (SVM), respectively. Contour variation features of salted sea cucumbers were extracted using a specific algorithm to improve the accuracy and stability of the model. Then, the dimensionality reduction and fusion of the feature images were achieved. According to the results of the models, the SVM identification model integrated with GLCM (GLCM-SVM) was found to be optimal, with accuracy, sensitivity, and specificity of $100 \%, 100 \%$, and $100 \%$, respectively. In particular, the sensitivity reached $100 \%$, demonstrating an excellent identification ability to excessively salted sea cucumbers of the optimized model. This study illustrated the potential for identification of salted sea cucumbers based on pressure contact by combining image texture of contour varying with machine learning.

\section{Introduction}

Sea cucumbers are rich in collagen, amino acids, trace elements, and a variety of bioactive substances and exhibit high medicinal value $[1,2]$. As fresh sea cucumbers are easily exposed to autolysis $[3,4]$, they are processed immediately by cooking, salting, or drying so that they can be conveniently stored and transported [5, 6]. Commercially available sea cucumbers mainly include dry sea cucumbers, salted sea cucumbers, and instant sea cucumbers. Salted sea cucumbers are not only used as raw material for dry sea cucumbers and instant sea cucumbers but also sold directly as the final product, which has a huge demand. The internal organs of fresh sea cucumbers are first removed, and then, they are cleaned, precooked, and salted to prepare salted sea cucumbers [7]. According to the Chinese Fisheries Industry Standard (SC/T3215-2014) regulations [8], the salt content of qualified salted sea cucumbers should be no more than $25 \%$. With the increase in salt content, the medicinal and nutritional values of sea cucumbers are reduced $[1,9]$. Additionally, texture 
property and flavor also decline [10-12]. Merchants illegally use saturated salt solution to repeatedly soak sea cucumbers to increase their weight and also mix unqualified products with excessive salt contents with qualified samples to make huge profits.

Currently, identification of unqualified salted sea cucumbers with excessive salt content is conducted using two methods: lab detection and artificial detection. Direct titration and potentiometric titration are the two commonly used lab detection methods for this purpose [13]. However, lab detection methods are time-consuming and destructive and have high requirements for professional personnel. Sea cucumbers are rich in collagen, the salt concentration interferes with the degree of protein denaturation, and the texture properties show complex variations $[1,14,15]$. Based on this, during the manufacturing of salted sea cucumbers, an artificial identification method is widely used to judge whether the salted sea cucumber has excessive salt content, by observing the dynamic recovery of the sea cucumbers after removing pressure contact. This method focuses primarily on texture properties, such as hardness and elasticity, requires high tester experience, is not conducive to largescale and automatic identification, and is easily affected by subjective factors of testers. The texture analyzer is a reliable device for the detection of texture properties and can achieve the quantitative detection of sample textures [16]. Wang et al. studied the influence of different heat treatment processes on the wall of sea cucumbers by means of a texture analyzer [17]. Geng et al. studied the relationship between the rehydration time and texture parameters such as hardness, chewiness, and rehydration rate using a texture analyzer [18]. Furthermore, in order to determine the structure and texture characteristics of meat products, a texture analyzer was used in parallel with other instruments to determine the characteristics of meat products [19]. However, the abovementioned method is destructive as it requires the material to be cut into sample blocks of standard specification, which takes a long time. The cucumber identification mainly focused on physical and chemical index laboratory tests, such as protein, polysaccharides, and fat $[20,21]$. The nondestructive testing method of unqualified salted sea cucumber identification has not been reported. Wang et al. have used hyperspectral technology to detect the freshness of sea cucumbers, which have provided some ideas for us to understand the characteristics of sea cucumber in nondestructive detection [22]. Therefore, an objective, rapid, nondestructive method of salted sea cucumbers is necessary to control and maintain the sea cucumber market and identify unqualified salted sea cucumbers.

The development of optical instrument and sensor technology has led to increasing application of computer vision technology for rapid and nondestructive food detections [23-25]. In recent years, a combination of machine learning and computer vision technology is popularly used to study the relationship between image feature information and detection targets, which has achieved good results in the evaluation of apparent meat quality, fat content and palatability prediction, automatic categorization of fruits, adulteration identification of food, and so forth [23, 26-28]. The above researches have proved that it is feasible to track the contour variation in the recovery process of salted sea cucumber with computer vision and machine learning. To sum up, the artificial identification method of observing the deformation during the recovery of sea cucumber after pressure contact has been proved to be rapid and effective, but it is not conducive to automated mass production. If existing instruments such as the texture analyzer mentioned above can be used to output uniform and standard pressure forces, and the contour variation can be tracked by a computer vision system, it is possible to realize the nondestructive and rapid identification of unqualified sea cucumbers.

This study is aimed at simulating the artificial identification method using a machine vision system and texture analyzer so as to establish a model reflecting the qualitative relationship between contour variation of salted sea cucumber during the recovery after pressure contact and its quality. The potential and feasibility of the aforementioned method for the identification of unqualified salted sea cucumbers were verified. The study was divided into five parts: (1) simulation of pressing in artificial identification and delivery of standard and uniform pressure forces using a texture analyzer, (2) development of an image acquisition system for capturing the contour images of salted sea cucumbers during the recovery after pressure contact, (3) establishment of an algorithm specifically for extracting contour images of salted sea cucumbers during the recovery and dimensionality reduction and fusion of contour information at the image level, (4) extraction of image textures based on histogram (HIS) and gray level cooccurrence matrices (GLCM), and (5) development of models for rapid and nondestructive identification of unqualified salted sea cucumbers. Figure 1 illustrates the identification process.

\section{Materials and Methods}

2.1. Materials and Method. Fresh sea cucumbers (100-120 g) were purchased from a local seafood market in Dalian, China, and transported to the lab in ice-containing insulation containers. After removing their organs and inner wall longitudinal ribs, they were cooked in a $100^{\circ} \mathrm{C}$ water bath for $30 \mathrm{~min}$ and stored at $4^{\circ} \mathrm{C}$ for use after natural cooling. The sea cucumbers were divided into two groups $[29,30]$ : the first group (G1) of samples were salted in a saturated salt solution for 2 days; the second group (G2) of samples were first salted in a supersaturated salt solution for 2 days and then salted in dry salt for 2 days. The salt solutions were changed every $24 \mathrm{~h}$.

2.2. Image Acquisition System. Figure 1(a) shows the image acquisition system for the identification of unqualified salted sea cucumbers. Its main components included a TMS-PRO texture analyzer (FTC, VA, USA), a camera (EOS 550C, Canon Inc., Tokyo, Japan), a rectangular probe, and an acrylic stage. Salted sea cucumber was placed on the stage, and pressure forces were applied on the sample with the texture analyzer. Contour variation images of the sample were collected during its recovery. The probe motion was divided into four phases, as shown in Figure 1(b): the probe was 


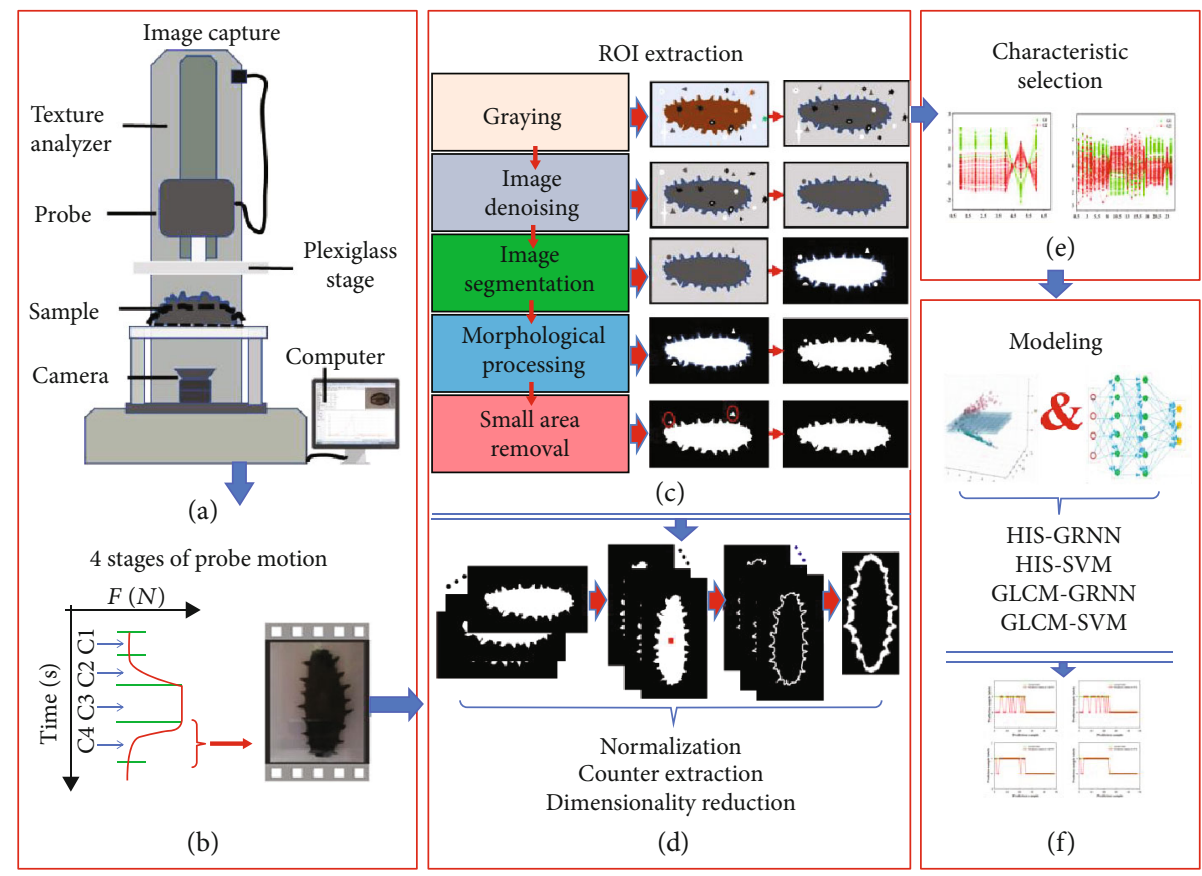

FIGURE 1: Identification process of unqualified salted sea cucumbers.

moved up without load motion (C1); the probe was loaded with pressure to the target force (C2); the probe was stationary and remained as the target force (C3); and the probe was quickly returned to its origin (C4). The test conditions of the texture analyzer were as follows: the test probe was a rectangular probe of $90 \times 160 \mathrm{~mm}^{2}$, no-load speed was $60 \mathrm{~mm} / \mathrm{min}$, trigger force was $0.2 \mathrm{~N}$, loading speed was $30 \mathrm{~mm} / \mathrm{min}$, target force was $60 \mathrm{~N}$, holding time was $12 \mathrm{~s}$, recovery speed was $990 \mathrm{~mm} / \mathrm{min}$, and return height was $50 \mathrm{~mm}$. The camera parameters were set as follows: the image resolution was $1280 \times 720$ pixel $^{2}$, frequency was $30 \mathrm{fps}$, and file format of the image was BMP.

2.3. Selection and Processing of Key-Frame Image (KI). The image sequences of 1-50 frames in phase $\mathrm{C} 4$ were determined as KIs, which were used to represent the contour variations of sea cucumbers during the recovery after pressure forces were revoked. As KIs had a large amount of data and interferences of noise, background, and other information, region of interest (ROI) extraction, ROI region normalization, ROI contour extraction, and KI dimensionality reduction and fusion (Figure 1(c)) were required to ensure accurate extraction of image features related to identification. The ROI extraction process used median filtering to eliminate isolated noise points in the image $[28,29]$, which applied a rectangular template with a $3 \times 3$ structure. The Otsu method was used to segment the sea cucumber from the background to obtain the binary image of the sea cucumber with the background removed [31]. Based on this, the binary image contour of the sea cucumber was smoothed: a closed operation was adopted to fuse the narrow gaps and slender curved parts of the sea cucumber contour to eliminate unconnected areas (small holes) and fill the gaps on the contour. A circular structural element with a radius of three pixels was selected to perform the closed operation; simultaneously, some small unconnected areas were removed. After morphological processing, the complete sea cucumber contour is obtained, but there are still some large connected areas in the image background, so small-area removal was used to eliminate larger noise; the removed area exceeded 3600 pixels, and the judgment principle of the connected area was eight neighborhoods. The final ROI obtained was the complete sea cucumber area $[32,33]$.

The interference of redundant information was reduced, and the variation characteristics of the contour were enhanced by fusing the ROI contour information of 50frame KIs into an image to achieve KI dimensionality reduction and fusion as follows. First, the ROI area of KI was standardized by Han and Bhanu's method [33] to ensure that the long axis of the sea cucumber was parallel to the horizontal position and all centroids coincided. Subsequently, based on this, the contour of the ROI region was extracted and the contour image sequences were fused in an image through logical addition operations to prepare for feature extraction. The logical operation process was as follows:

$$
\begin{aligned}
& A_{i}(x, y)=\sum_{1}^{N} B_{i}(x, y), \\
& O(\mathrm{x}, \mathrm{y})=\left\{\begin{array}{l}
0, A_{i}(\mathrm{x}, \mathrm{y})=0, \\
1, B_{i}(x, y)>0,
\end{array}\right.
\end{aligned}
$$

where $A_{i}(x, y)$ is the pixel of the dimensionality-reduced image, $B_{i}(x, y)$ is the pixel of binary KI, $N=50$ reflects the sequence number of $\mathrm{KI}$, and $O(x, y)$ is the feature image after dimensionality reduction. 
2.4. Feature Extraction. Image texture is one of the most important inherent features of an image, which has important statistical significance in representing material properties [34-36]. The image texture can be regarded as a function of spatial variation of the brightness intensity of the images. Through the specific image texture features, image characteristics such as smoothness, coarseness, and regularity of each surface in different order directions can be measured [37]. After fusing the contour features of KIs into an image, image texture features were extracted to represent time-varying contour features during the recovery stage (C4) of the samples. At present, a large number of statistical image texture features have been applied, which obtained good results in industrial applications, biomedical, remote sensing areas, and target recognition. Among many, HIS and GLCM have been widely used in texture analysis and classification [38]. Therefore, HIS and GLCM methods were then used to extract image texture features in the study [39, 40]. Mean $(m)$, standard variation $(\sigma)$, smoothness $(R)$, third-order matrix $(\mu)$, uniformity $(U)$, and entropy $(e)$ were extracted as the HIS-based image texture features. Thereinto, $m, \sigma, R$, and $e$ represent the average brightness, average contrast, average smoothness of the brightness, and randomness in the ROI area, respectively. $\mu$ represents the skewness of the HIS; if the HIS is symmetrical, the value is 0 ; if skewed to the right, the value is positive; and if skewed to the left, the value is negative. $U$ represents the difference between gray levels, which was the largest when the gray levels were equal. The formulas for the features were shown below:

$$
\begin{aligned}
p\left(z_{i}\right) & =\frac{z_{i}}{N}, \quad i=0,1,2, \cdots, L-1, \\
m & =\sum_{i=0}^{L-1} z_{i} p\left(z_{i}\right), \\
\sigma & =\sqrt{\sum_{i=0}^{L-1}\left(z_{i}-m\right)^{2} p\left(z_{i}\right),} \\
R & =1-\frac{1}{\left(1+\sigma^{2}\right)}, \\
\mu & =\sum_{i=0}^{L-1}\left(z_{i}-m\right)^{3} p\left(z_{i}\right), \\
U & =\sum_{i=0}^{L-1} p^{2}\left(z_{i}\right), \\
e & =-\sum_{i=0}^{L-1} p\left(z_{i}\right) \log _{2} p\left(z_{i}\right),
\end{aligned}
$$

where the feature image obtained in this study was a binary matrix with a grayscale of 2 and a gray level of 0 or $1 ; p\left(z_{i}\right)$ is the mathematical expression of HIS; $z_{i}$ refers to the number of pixels with a gray level of $i$; $N$ refers to the number of pixels corresponding to the image; and $L$ refers to the grayscale. GLCM was defined as the probability that two pixels with gray levels $i$ and $j$ were located at points with a distance of $d$ ( 1 pixel) in the $\theta$ direction $\left(0^{\circ}, 45^{\circ}, 90^{\circ}\right.$, and $\left.135^{\circ}\right)$. It was denoted by $p(i, j ; d, \theta)\left(p_{i j}\right)$. Contrast $(C)$, correlation (Cor), energy $(E)$, homogeneity $(H)$, maximum probability $\left(P_{\max }\right)$, and entropy (En) were extracted as the GLCM-based image texture features. Thereinto, $C$ is a measure of the drastic change between the adjacent pixels. When the image has an almost constant grayscale, the $C$ value is lower. Cor is a value to measure the linear dependence of the image grayscale. It presents the linear relationship between the grayscales of neighbouring pixel pairs. $E$ is a measure of the uniformity or thickness of the texture. When all the elements of GLCM are almost equal, it achieves the smallest value. $H$ measures the homogeneity of the image, which is sensitive to the near-diagonal elements of the GLCM, indicating the grayscale similarity between adjacent pixels. $P_{\max }$ measures the maximum value of $p_{i j}$. En measures the disorder of an image, which is negatively correlated with E. [41-43]. A total of twenty-four image texture features were obtained based on GLCM, which were $C$, Cor, $E, H, P_{\max }$, and En in four directions $\left(0^{\circ}, 45^{\circ}, 90^{\circ}\right.$, and $\left.135^{\circ}\right)$ of the image. The GLCM-based image texture features were expressed as follows [39, 44]:

$$
\begin{aligned}
C & =\sum_{i=1}^{k} \sum_{j=1}^{k}(i-j)^{2} p_{i j}, \\
\text { Cor } & =\sum_{i=1}^{k} \sum_{j=1}^{k} \frac{\left(i-m_{r}\right)\left(j-m_{c}\right) p_{i j}}{\sigma_{r} \sigma_{c}}\left(\sigma_{r} \neq 0, \sigma_{c} \neq 0\right), \\
E & =\sum_{i=1}^{k} \sum_{j=1}^{k} p_{i j}^{2}, \\
H & =\sum_{i=i}^{k} \sum_{j=1}^{k} \frac{p_{i j}^{2}}{1+|i-j|}, \\
P_{\max } & =\max _{i, j}\left(p_{i j}\right), \\
\text { En } & =-\sum_{i=1}^{k} \sum_{j=1}^{k} p_{i j} \log _{2} p_{i j},
\end{aligned}
$$

where $k$ is the order of GLCM, with $k=2$ as the image that is a binary image; $m_{r}$ and $m_{c}$ represent the mean of row and column for GLCM; and $\sigma_{r}$ and $\sigma_{c}$ are line and column standard deviation (SD) of GLCM. Variance normalization was performed on the extracted features to remove the influence of different feature dimensions [28]:

$$
z_{n}=\frac{x-u_{n}}{\sigma_{n}},
$$

where $z_{n}$ is the feature value after variance normalization, $x$ is the SD before variance normalization, $u_{n}$ is the mean of the feature value, and $\sigma_{n}$ is the $\mathrm{SD}$ of the feature value.

2.5. Identification Model of Unqualified Salted Sea Cucumbers. As a radial neural network based on nonlinear regression analysis, general regression neural networks 
(GRNN) showed good nonlinear mapping capability and global 1 convergence, which was widely applied in classification, detection, and prediction [45]. By introducing the kernel function mapping method, nonlinear problems were transformed into linear problems in the high-dimensional space in using a support vector machine (SVM), which had obvious advantages in terms of solving nonlinear problems, small samples, and high-dimensional detection [46, 47]. Therefore, GRNN and SVM were employed to establish models for the identification of unqualified salted sea cucumbers in this study. A total of 120 samples were used to establish the adulteration model, with 60 samples in G1 and G2 groups, respectively. The ratio of the training set to the test set was $6: 4$, that is, 72 samples were selected for the training set of the model ( $\mathrm{G} 1=36$ and $\mathrm{G} 2=36)$ and 48 samples were selected for the model prediction set $(\mathrm{G} 1=24$ and $\mathrm{G} 2=24)$. Neurons of the input layer in the GRNN and SVM models were composed of image texture features [44]. The one-way analysis of variance (ANOVA) was used to eliminate the features that were not significantly affected by salt content change. In this study, the HIS-based detection model had six inputs, which corresponded to $m, s, R, \mu, U$, and $e$ image texture features. The GLCM-based detection model had 22 inputs, which corresponded to the GLCM image texture features $C\left(45^{\circ}, 90^{\circ}\right.$, and $\left.135^{\circ}\right)$, Cor $\left(0^{\circ}, 45^{\circ}, 90^{\circ}\right.$, and $\left.135^{\circ}\right)$, $E\left(0^{\circ}, 45^{\circ}, 90^{\circ}\right.$, and $\left.135^{\circ}\right), H\left(45^{\circ}, 90^{\circ}\right.$, and $\left.135^{\circ}\right), P_{\max }\left(0^{\circ}\right.$, $\left.45^{\circ}, 90^{\circ}, 135^{\circ}\right)$, and $\operatorname{En}\left(0^{\circ}, 45^{\circ}, 90^{\circ}\right.$, and $\left.135^{\circ}\right)$. The outputs were the corresponding category labels; G1 corresponded to label 1 and G2 to label 2, which, respectively, represented qualified samples (salt content $<25 \%$ ) and unqualified samples (salt content $>25 \%$ ).

The spread value (SV) of GRNN and penalty parameter $(c)$ and kernel function $(g)$ of SVM are very critical for model accuracy and generalized ability. These parameters must be optimized and determined before models are built. Sixfold cross-validation $(\mathrm{CV})$ is applied in this paper to evaluate the accuracy and generalized ability of the GRNN model with different SV. The search range of the SV is set to 0.1 to 2 , and the step length is set to 0.1 . The results of the GRNN model corresponding to different SV were analyzed by means of the mean square error (MES), and the SV was determined as the optimal after sixfold CV with the minimum MES $[48,49]$. For the SVM model, the radial basis function was selected as the kernel function. Through the sixfold cross-validation, the optimization of parameters of $c$ and $g$ is realized by using the genetic algorithm here, in which the number of iterations, the population, and the search range of $c$ and $g$ were set to $200,20,2^{-10}-2^{10}$, and $2^{-10}-2^{10}$, respectively [50].

According to the method of Wang et al. for model performance evaluating [51], the performance of the model was evaluated using parameters such as accuracy (Ac), sensitivity $(\mathrm{Se})$, and specificity (Sp), where Ac indicated the ratio of the number of the salted cucumber samples that are identified correctly to the total number of samples irrespective of the sample type; Se and Sp represented the proportion of correctly identified qualified samples and unqualified samples, respectively. True positive (TP) and true negative (TN) indicate correct identification of a qualified and unqualified
TABLE 1: Statistical data of the salted sea cucumber samples.

\begin{tabular}{lcccc}
\hline $\begin{array}{l}\text { Different groups } \\
\text { of samples }\end{array}$ & $\begin{array}{c}\text { Number of } \\
\text { samples }\end{array}$ & $\max (\%)$ & $\min (\%)$ & SD \\
\hline G1 & 60 & 20.34 & 18.60 & 0.33 \\
G2 & 60 & 26.00 & 29.32 & 0.23 \\
\hline
\end{tabular}

max, min, and SD represent the maximum, minimum, and standard deviation of salinity in different groups of salted sea cucumber samples, respectively.

salted sea cucumber sample; false positive (FP) indicates that the unqualified salted sea cucumber was identified as a qualified sample; false negative (FN) indicates that the qualified sample was identified as an unqualified one. The parameters (Ac, Se, and Sp) were calculated as follows:

$$
\begin{aligned}
& \mathrm{Ac}=\frac{\mathrm{NTP}+\mathrm{NTN}}{\mathrm{NTP}+\mathrm{NTN}+\mathrm{NFP}+\mathrm{NFN}} \times 100 \%, \\
& \mathrm{Sp}=\frac{\mathrm{NTN}}{\mathrm{NTN}+\mathrm{NFP}} \times 100 \%, \\
& \mathrm{Se}=\frac{\mathrm{NTP}}{\mathrm{NTP}+\mathrm{NFN}} \times 100 \%,
\end{aligned}
$$

where NTP, NTN, NFP, and NFN indicate the number of TP, TN, FP, and FN samples, respectively. The higher the parameter value, the better the detection effect of the model for qualified and unqualified salted sea cucumber samples. The Matlab 2014b (The MathWorks Inc., MA, USA) software was used for the processing of image data and construction of the detection model.

\section{Results and Discussion}

3.1. Sample Statistical Analysis. The statistical data of G1 and G2 samples are shown in Table 1. A total of 120 samples with different contents of sea salt were used for experiments. The G1 samples with salt contents less than $25 \%$ were defined as qualified, while G2 samples with salt contents more than $25 \%$ were defined as unqualified.

3.2. ROI and Its Contour Extraction. The quality of ROI extraction directly affected the accuracy of feature extraction, which was one of the most important steps in image analysis and discrimination $[23,52]$. Figure 2 shows the results of KI processing. After graying and median filtering (Figures 2 (b) and 2(c)), some noise was eliminated, and the image quality was improved to a certain extent. As shown in Figure 2(d), the image segmented by the Otsu method removed most of the background information interference [53]; however, contours of sea cucumbers appeared to have pits and spikes. As shown in Figure 2(e), the image contour was smoothed to some extent after the closed operation, eliminating the impact on contours due to light and acquisition equipment. Then, the small-area removal method was used to remove a small amount of large-area noise image contour (Figure 2(f)) to obtain the final image of the ROI region and provide a better image environment for subsequent feature extraction. Finally, standardized processing was implemented, and the 


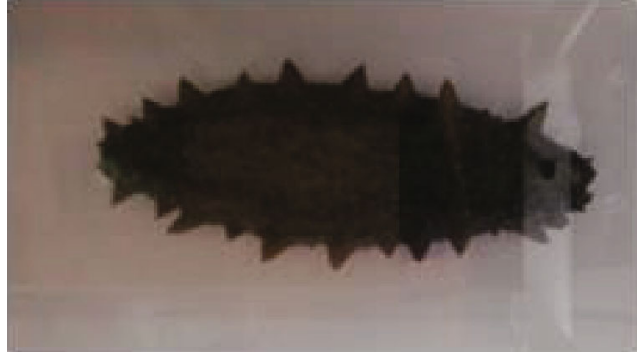

(a)

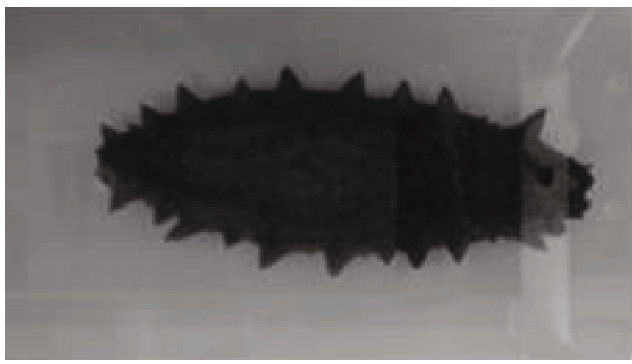

(c)

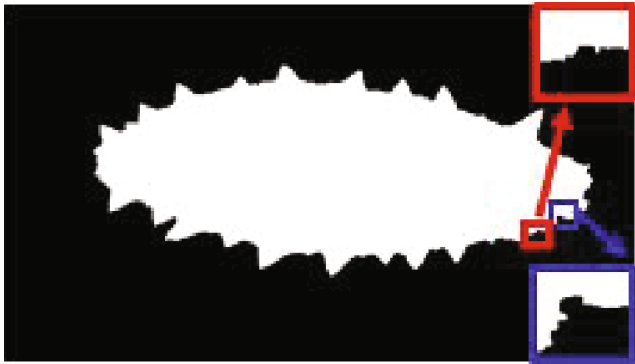

(e)

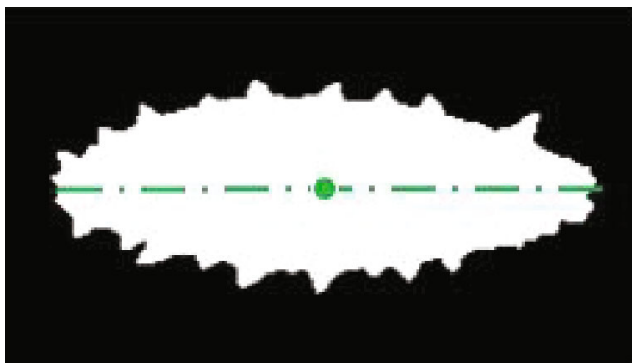

(g)

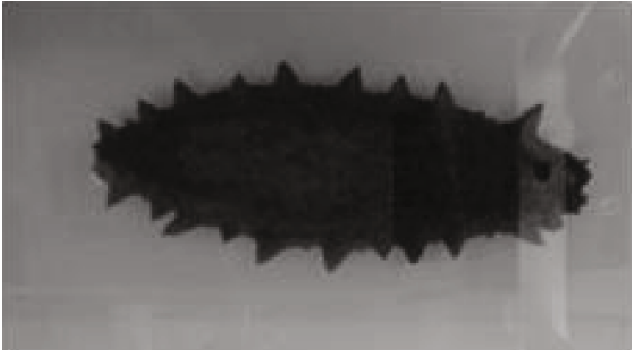

(b)

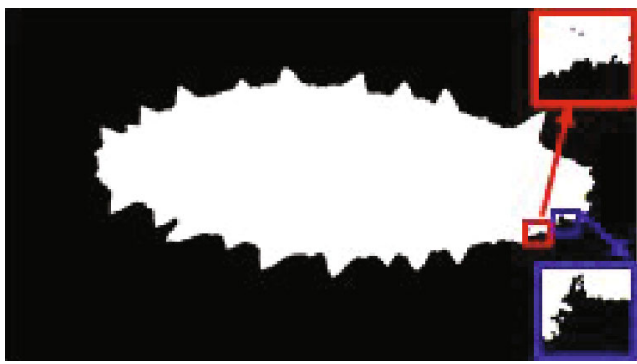

(d)

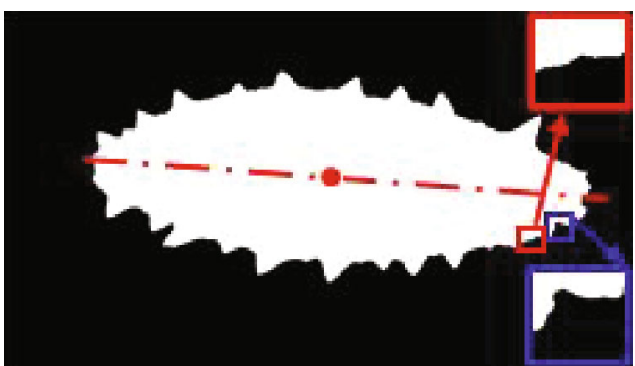

(f)

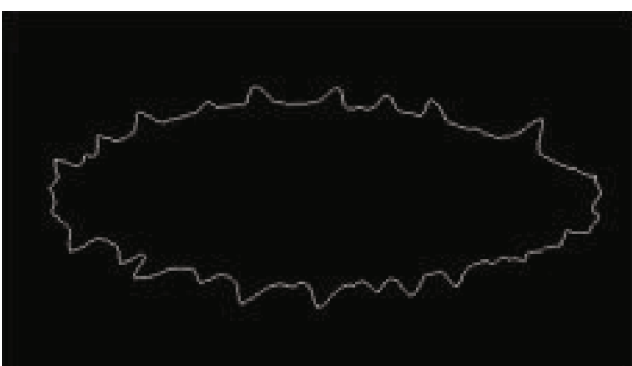

(h)

Figure 2: Processing of target images.

centroid and axial normalization of KI was realized to ensure that the sea cucumber contour during the recovery was more comparable, as shown in Figure $2(\mathrm{~g})$. On this basis, the ROI region contour (Figure $2(\mathrm{~h})$ ) was extracted to eliminate the effect of collection position and angle on contour variation, which was prepared for the logical addition operation of 50 KIs.

3.3. Analysis of the Feature Images and the Microstructure Images. The contour variation information during the recovery of sea cucumber in the $\mathrm{C} 4$ stage was fused into one image, which was defined as the feature image, so as to reduce the amount of calculation and the interference of redundant information through the logical addition opera- tions of 50 contour images (Figures 3(a) and 3(c)). One of the feature images of G1 and G2 samples is demonstrated in Figures 3(a) and 3(c), respectively. The external contour was the sea cucumber contour of the first image in the $\mathrm{C} 4$ stage, and the internal contour was the sea cucumber contour of the 50th image. After salting in saturated saline, the collagen denatured and the fiber structure was damaged. As shown in Figure 3(d), compared with the G1 sample (Figure 3(b)), the filamentous fibers of the G2 sample disappeared, and the fiber section showed a flaky structure and was more likely to break. The gap of the G2 sample became larger, causing easy exchange between the body wall of sea cucumber and the salt of the environment. The microstructure images indicated that the texture properties of the salted sea 


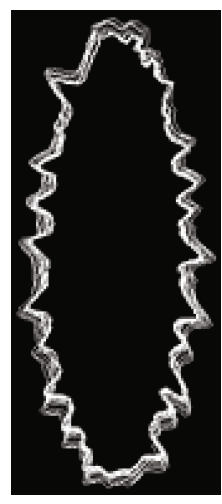

(a)

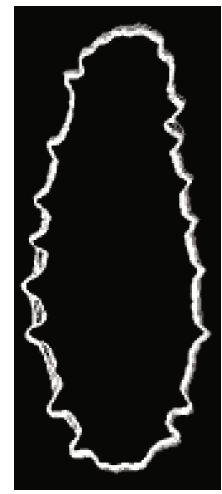

(c)

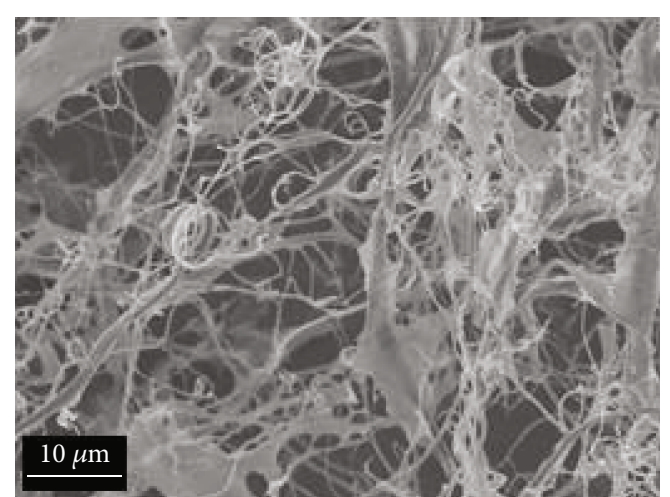

(b)

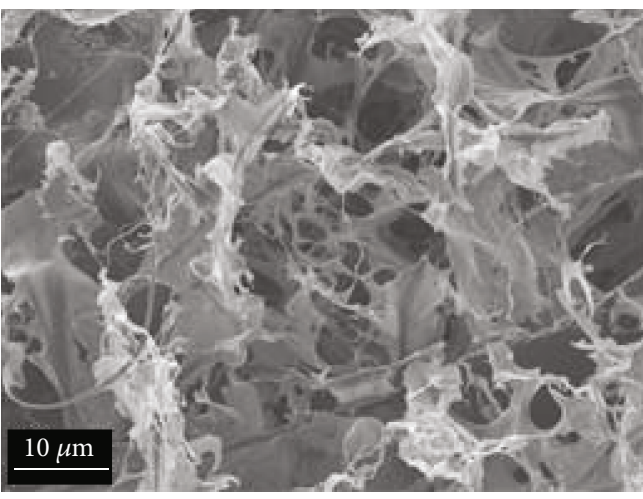

(d)

Figure 3: Feature image and microstructure image of salted sea cucumber samples. (a) and (c) are the randomly selected feature image in G1 and $\mathrm{G} 2$; (b) and (d) are the corresponding microstructure images, respectively.

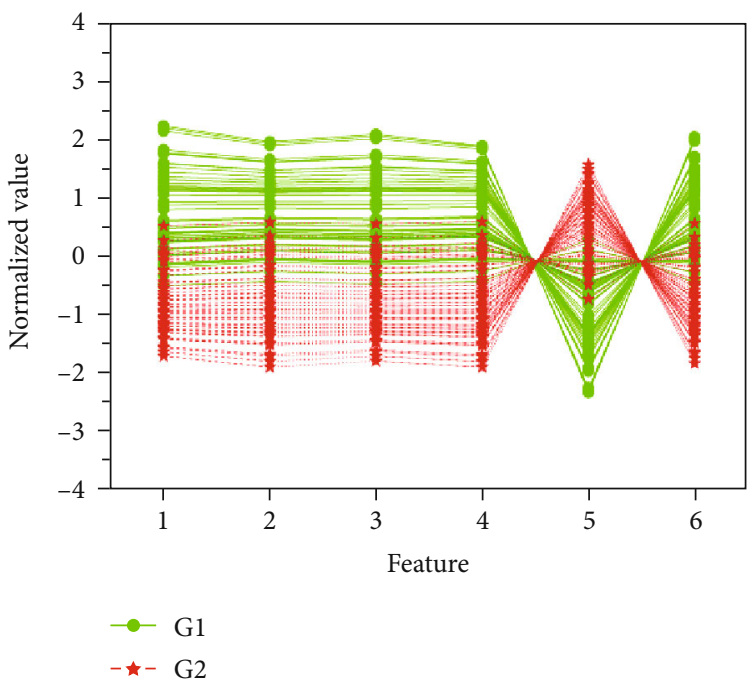

(a)

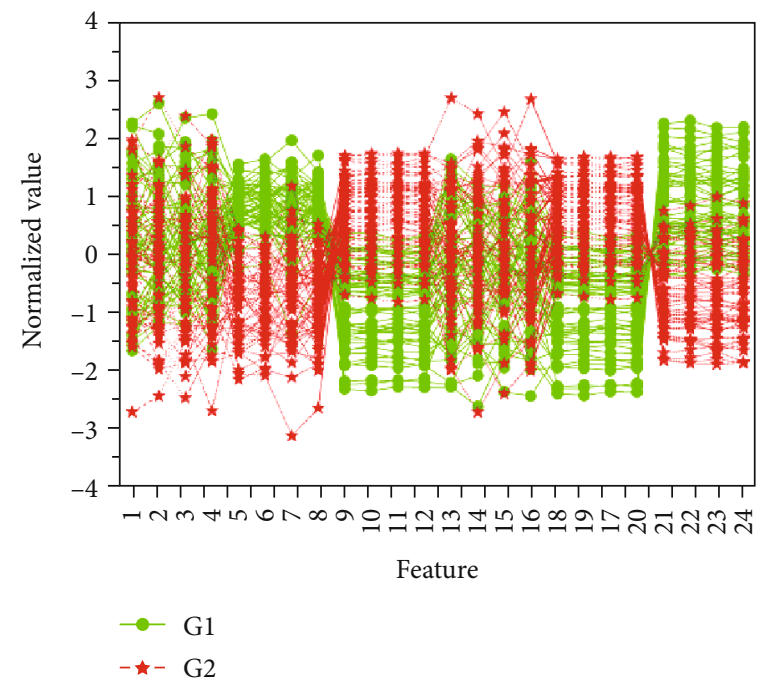

(b)

FIGURE 4: Image texture feature distribution: (a) the scatter plot of HIS-based image texture feature distribution; features 1-6 are $m, s, R, \mu, c$, and $e$. (b) The scatter plot of image texture distribution based on GLCM; features 1-4, 5-8, 9-12, 13-16, 17-20, and 21-24 represent six image texture features $C$, Cor, $E, H, P_{\max }$, and En, respectively, and the corresponding angles were $0^{\circ}, 45^{\circ}, 90^{\circ}$, and $135^{\circ}$.

cucumber may change complicated with the salt content. Guizani et al. studied the effect of salting treatment on the quality of shark meat. The study demonstrated that the num- ber of salting days and salt content reached a certain level, the hardness of shark meat increased, the elasticity decreased, and the cohesiveness increased [54]. As shown in 
Figures 3(a) and 3(c), under the same target force, the deformation of the G1 sample was less than that of the G2 sample, which might have been caused by the increase in hardness with the increase in the salt content of G2 sample. To sum up, with the increase in the salt content, the hardness, elasticity, and other features of salted sea cucumber samples changed completely, resulting in a difference in deformation degree and recovery speed between G1 and G2 samples. Therefore, in this study, image texture features were used to characterize the contour variation in the recovery process, and the relationship between image texture features and salt content was established to realize the identification of unqualified salted sea cucumber.

3.4. Extraction and Analysis of Image Features. Figure 4 shows a scatter plot based on the HIS and GLCM image texture feature. Figure 4(a) shows that the distributions of six HIS-based image texture features $(m, s, R, \mu, U$, and $e$ ) were significantly different. One-way ANOVA was performed on G1 and G2 samples. The results are shown in Table 2. The six image texture features of G1 and G2 samples were all significantly related, indicating that the impact of salt content on the aforementioned image texture features was extremely significant. Figure 4(b) shows that, among the GLCM-based image texture features, differences between the $C$ and $H$ features of G1 and G2 samples were small, and the differences among Cor, $E, P_{\max }$, and En were obvious. The significant analysis results of G1 and G2 samples are shown in Table 3. No significant difference was found between image texture feature $C$ and $H$ at $0^{\circ}$, indicating that the salt content had no significant effect on the aforementioned features. Extremely significant differences were found among Cor, $E$, $P_{\max }$, and En of G1 and G2 samples, indicating that contour similarity, local change, and uniformity under the different salting processes showed significant variations during the recovery process of the samples. Based on the aforementioned analysis, $m, s, R, \mu, U, e, C\left(45^{\circ}, 90^{\circ}\right.$, and $\left.135^{\circ}\right), H$ $\left(45^{\circ}, 90^{\circ}\right.$, and $\left.135^{\circ}\right)$, Cor $\left(0^{\circ}, 45^{\circ}, 90^{\circ}\right.$, and $\left.135^{\circ}\right), E\left(0^{\circ}, 45^{\circ}\right.$, $90^{\circ}$, and $\left.135^{\circ}\right), P_{\max }\left(0^{\circ}, 45^{\circ}, 90^{\circ}\right.$, and $\left.135^{\circ}\right)$, and En $\left(0^{\circ}, 45^{\circ}\right.$, $90^{\circ}$, and $135^{\circ}$ ) were selected for modeling.

3.5. Model Development. According to the aforementioned research results and image texture features during the recovery process, GRNN and SVM were used to establish detection models for the unqualified salted sea cucumber identification. The models based on HIS and GLCM features were named HIS-GRNN, GLCM-GRNN, HIS-SVM, and GLCMSVM, respectively.

Figure 5 shows the SV optimization process of sixfold $\mathrm{CV}$. For each $\mathrm{CV}$ process, with the increase of SV, most MES values showed an overall trend of first decrease and then increase, which indicates the value of SV has a certain influence on the performance of GRNN models. As shown in Figure 5, the minimum MSE values for HIS-GRNN and GLCM-GRNN models were obtained during the 1st and 2nd CV, respectively; accordingly, 0.2 and 0.9 were determined to be the optimized SVs for HIS-GRNN and GLCM-GRNN modeling.
TABLE 2: Image texture feature statistics of G1 and G2 samples based on HIS.

\begin{tabular}{lccc}
\hline Feature & $\begin{array}{c}\text { Mean value of } \\
\text { G1 samples }\end{array}$ & $\begin{array}{c}\text { Mean value of } \\
\text { G2 samples }\end{array}$ & $P$ \\
\hline$m$ & $0.78 \pm 0.68$ & $-0.78 \pm 0.55$ & $1.88 \times 10^{-26 * *}$ \\
$s$ & $0.79 \pm 0.59$ & $-0.79 \pm 0.62$ & $2.32 \times 10^{-27 * *}$ \\
$R$ & $0.79 \pm 0.64$ & $-0.79 \pm 0.59$ & $4.51 \times 10^{-27 * *}$ \\
$\mu$ & $0.79 \pm 0.58$ & $-0.79 \pm 0.63$ & $1.09 \times 10^{-27 * *}$ \\
$U$ & $-0.79 \pm 0.65$ & $0.79 \pm 0.57$ & $7.31 \times 10^{-27 * *}$ \\
$E$ & $0.79 \pm 0.62$ & $-0.79 \pm 0.60$ & $3.33 \times 10^{-27 * *}$ \\
\hline
\end{tabular}

$P$ means the measured significance level. ${ }^{*} P<0.05$ means significant difference at 0.05 levels. ${ }^{* *} P<0.01$ means significant difference at 0.01 levels.

TABLE 3: Image texture feature statistics of G1and G2 samples based on GLCM.

\begin{tabular}{|c|c|c|c|c|}
\hline Feature & Direction & $\begin{array}{l}\text { Mean value of } \\
\text { G1 samples }\end{array}$ & $\begin{array}{l}\text { Mean value of } \\
\text { G2 samples }\end{array}$ & $P$ \\
\hline \multirow{4}{*}{ C } & $0^{\circ}$ & $0.16 \pm 1.03$ & $-0.16 \pm 0.95$ & $7.26 \times 10^{-2}$ \\
\hline & $45^{\circ}$ & $0.23 \pm 0.89$ & $-0.23 \pm 1.06$ & $1.21 \times 10^{-2 *}$ \\
\hline & $90^{\circ}$ & $0.26 \pm 0.86$ & $-0.26 \pm 1.07$ & $0.47 \times 10^{-2 * *}$ \\
\hline & $135^{\circ}$ & $0.20 \pm 0.85$ & $-0.20 \pm 1.10$ & $3.15 \times 10^{-2 *}$ \\
\hline \multirow{4}{*}{ Cor } & $0^{\circ}$ & $0.84 \pm 0.34$ & $-0.84 \pm 0.69$ & $5.52 \times 10^{-33 * *}$ \\
\hline & $45^{\circ}$ & $0.86 \pm 0.35$ & $-0.86 \pm 0.63$ & $1.51 \times 10^{-36 * *}$ \\
\hline & $90^{\circ}$ & $0.71 \pm 0.56$ & $-0.71 \pm 0.83$ & $1.04 \times 10^{-19 * *}$ \\
\hline & $135^{\circ}$ & $0.82 \pm 0.37$ & $-0.82 \pm 0.71$ & $2.08 \times 10^{-31 * *}$ \\
\hline \multirow{4}{*}{$E$} & $0^{\circ}$ & $-0.77 \pm 0.68$ & $0.77 \pm 0.59$ & $7.58 \times 10^{-25 * *}$ \\
\hline & $45^{\circ}$ & $-0.76 \pm 0.68$ & $0.76 \pm 0.61$ & $2.97 \times 10^{-24 * *}$ \\
\hline & $90^{\circ}$ & $-0.77 \pm 0.66$ & $0.77 \pm 0.61$ & $4.36 \times 10^{-25 * *}$ \\
\hline & $135^{\circ}$ & $-0.76 \pm 0.68$ & $0.76 \pm 0.61$ & $2.11 \times 10^{-24 * *}$ \\
\hline \multirow{4}{*}{$H$} & $0^{\circ}$ & $-0.16 \pm 1.03$ & $0.16 \pm 0.95$ & $7.26 \times 10^{-2}$ \\
\hline & $45^{\circ}$ & $-0.23 \pm 0.89$ & $0.23 \pm 1.06$ & $1.21 \times 10^{-2 *}$ \\
\hline & $90^{\circ}$ & $-0.26 \pm 0.86$ & $0.26 \pm 1.07$ & $4.71 \times 10^{-3 * *}$ \\
\hline & $135^{\circ}$ & $-0.20 \pm 0.85$ & $0.20 \pm 1.10$ & $3.15 \times 10^{-2 *}$ \\
\hline \multirow{4}{*}{$P_{\max }$} & $0^{\circ}$ & $-0.77 \pm 0.71$ & $0.77 \pm 0.57$ & $1.07 \times 10^{-24 * *}$ \\
\hline & $45^{\circ}$ & $-0.76 \pm 0.70$ & $0.76 \pm 0.59$ & $3.64 \times 10^{-24 * *}$ \\
\hline & $90^{\circ}$ & $-0.77 \pm 0.69$ & $0.77 \pm 0.59$ & $5.8 \times 10^{-25 * *}$ \\
\hline & $135^{\circ}$ & $-0.76 \pm 0.70$ & $0.76 \pm 0.59$ & $2.68 \times 10^{-24 * *}$ \\
\hline \multirow{4}{*}{ En } & $0^{\circ}$ & $0.75 \pm 0.68$ & $-0.75 \pm 0.64$ & $2.26 \times 10^{-23 * *}$ \\
\hline & $45^{\circ}$ & $0.74 \pm 0.66$ & $-0.74 \pm 0.67$ & $1.12 \times 10^{-22 * *}$ \\
\hline & $90^{\circ}$ & $0.75 \pm 0.64$ & $-0.75 \pm 0.68$ & $3.28 \times 10^{-23 * *}$ \\
\hline & $135^{\circ}$ & $0.75 \pm 0.66$ & $-0.75 \pm 0.67$ & $6.81 \times 10^{-23 * *}$ \\
\hline
\end{tabular}

$P$ means measured significance level. ${ }^{*} P<0.05$ means significant difference at 0.05 levels. ${ }^{* *} P<0.01$ means significant difference at 0.01 levels. 


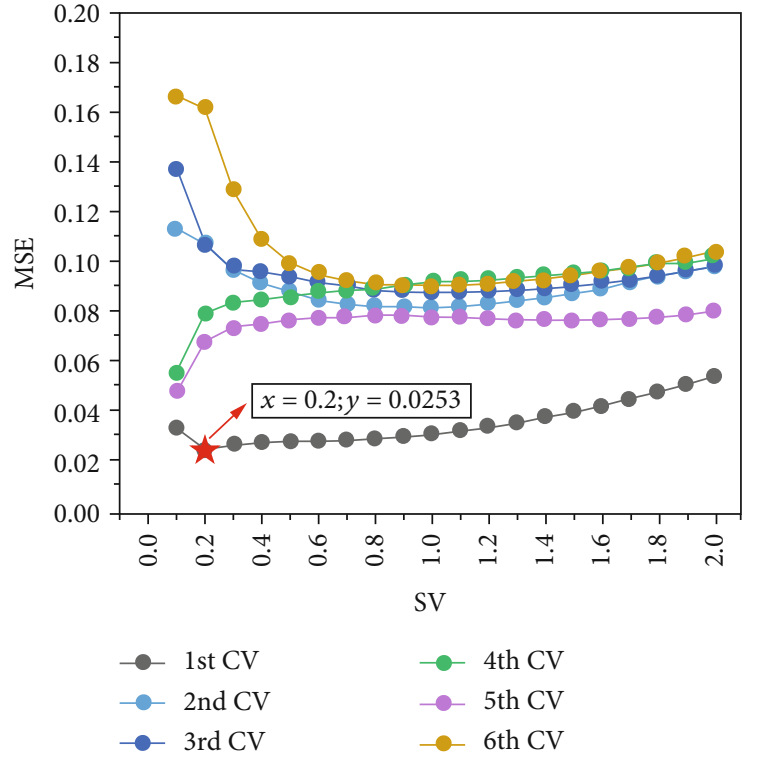

(a)

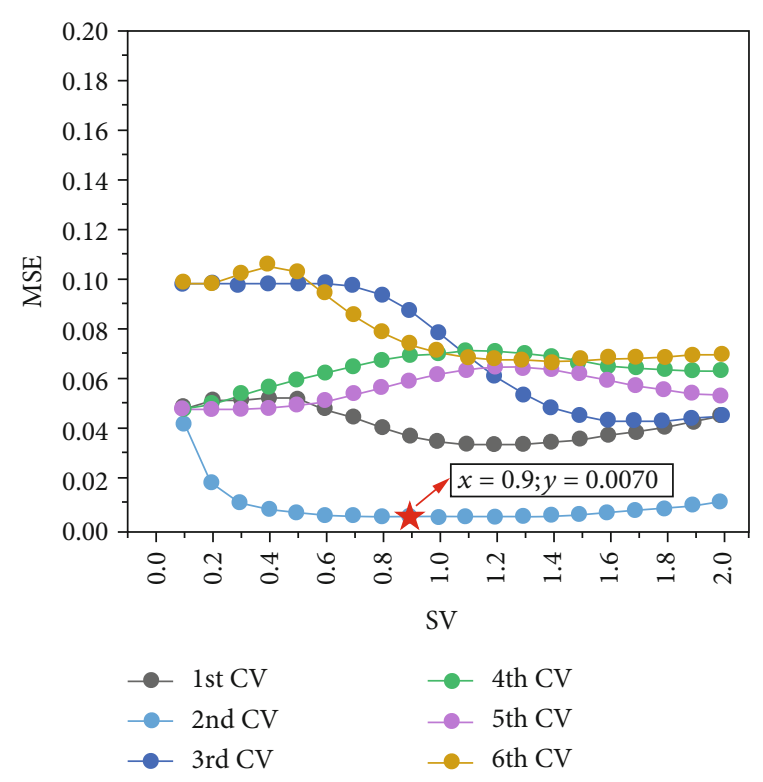

(b)

FIGURE 5: Six times optimization process for SV of HIS-GRNN (a) and GLCM-GRNN (b) models.

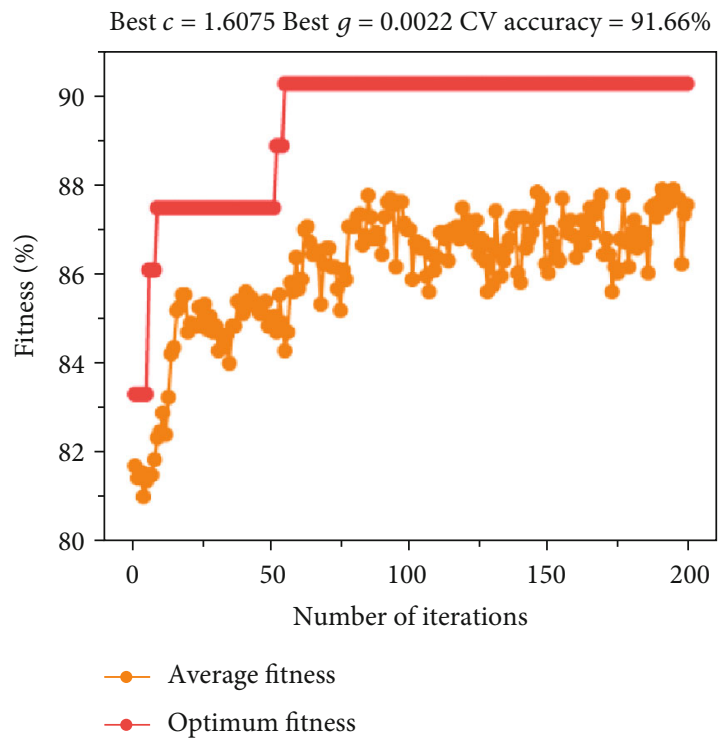

(a)

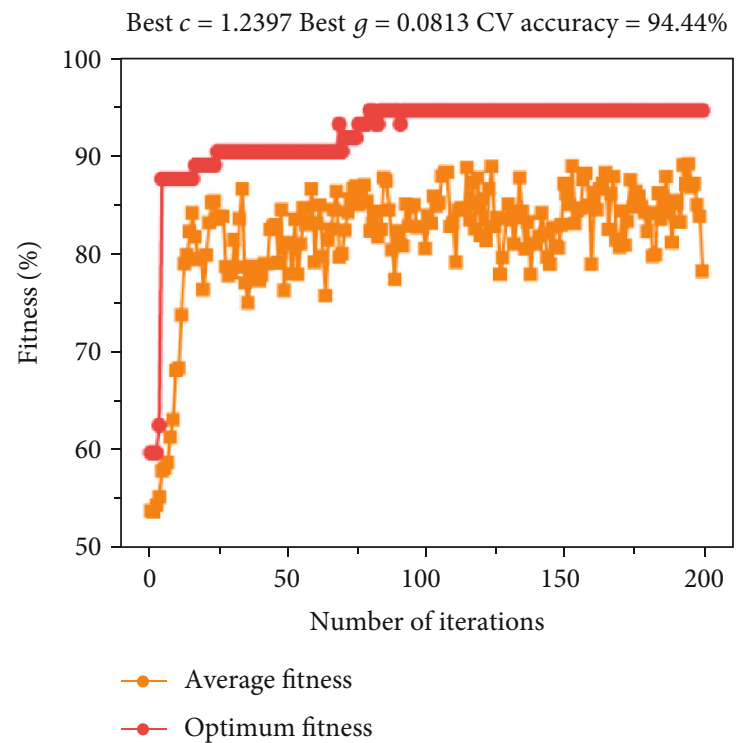

(b)

Figure 6: Optimization fitness curve of HIS-SVM (a) and GLCM-SVM (b) models.

Figure 6 shows the parameters $c$ and $g$ of the HIS-SVM and GLCM-SVM model optimization fitness curve. In order to avoid overfitting or underfitting, the highest accuracy of fivefold $\mathrm{CV}$ of its training set is taken as the fitness function for parameters evaluating. As shown in Figure 6, the orange circle and the red dots represent the average fitness and the best fitness of each iteration, respectively. When the best fitness reaches the maximum, the corresponding value of $c$ and $g$ is determined as the optimal parameters, that is, when the highest accuracy of fivefold CV of the training set was $91.66 \%$ and $94.44 \%$, the optimized parameters $c$ and $g$ of
TABLE 4: The performances of the models.

\begin{tabular}{lccccc}
\hline Classifier & $\begin{array}{c}\text { No. of } \\
\text { inputs }\end{array}$ & Ac (\%) & Se (\%) & Sp (\%) & $\begin{array}{c}\text { Operation } \\
\text { time }(\mathrm{s})\end{array}$ \\
\hline HIS-GRNN & 6 & 85.42 & 95.83 & 70.83 & 0.1575 \\
GLCM- & 22 & 95.83 & 100 & 91.66 & 0.1366 \\
GRNN & 6 & 83.33 & 66.66 & 100 & 0.0830 \\
HIS-SVM & 22 & 100 & 100 & 100 & 0.0846 \\
GLCM-SVM & & & & &
\end{tabular}




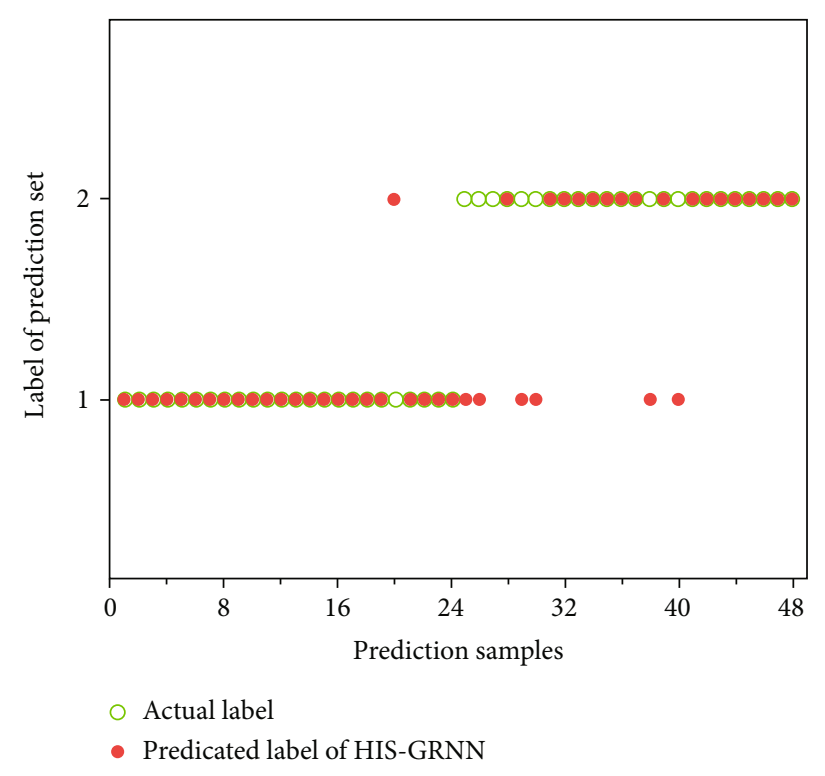

(a)

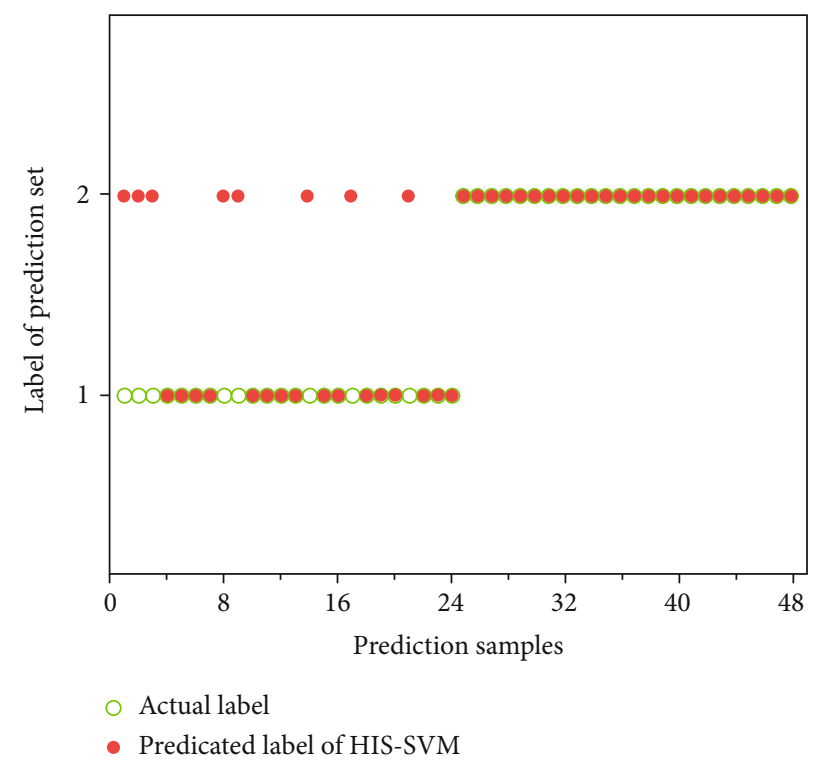

(c)

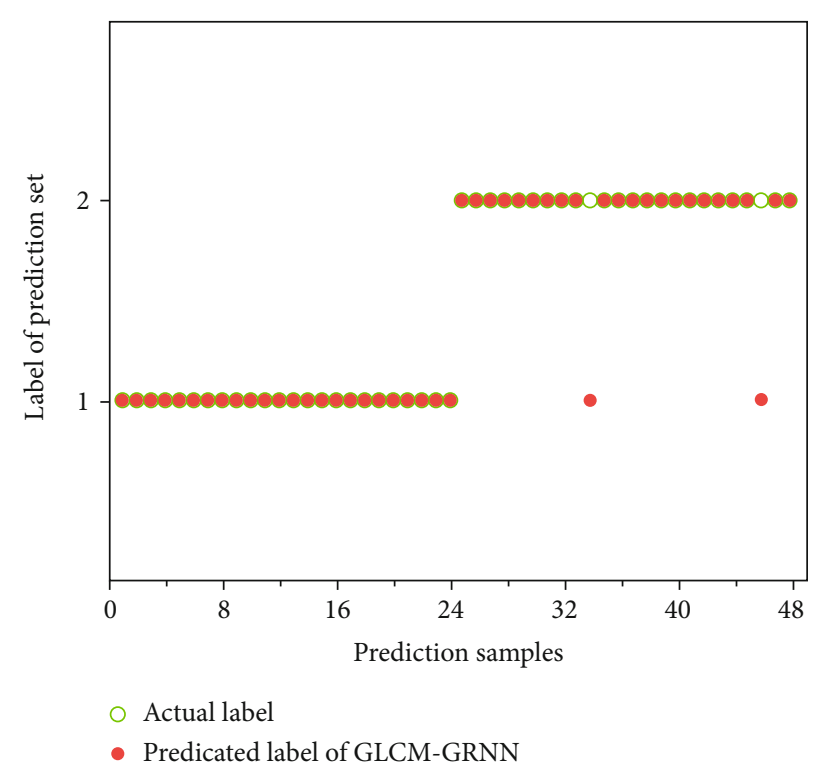

(b)

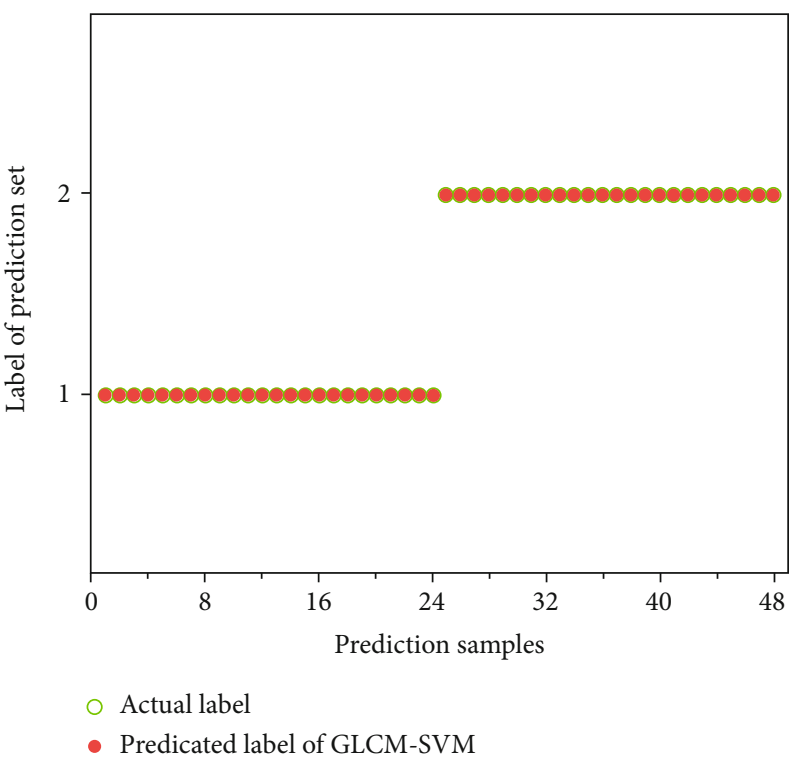

(d)

FIGURE 7: Identified results of the (a) HIS-GRNN, (b) GLCM-GRNN, (c) HIS-SVM, and (d) GLCM-SVM models.

HIS-SVM were determined as 1.6075 and 0.0022 , and the parameters of the GLCM-SVM model were determined as 1.2397 and 0.0813 , respectively.

Table 4 shows the performances of the four models: HISGRNN, GLCM-GRNN, HIS-SVM, and GLCM-SVM. For the prediction set, the identification results are intuitively illustrated in Figure 7. The operation times of all GRNN models were greater than that of SVM models, which indicated that the SVM model was faster in handling the classification of salted sea cucumber. In this study, the number of input had a small effect on the modeling operation time, but the advantages of the HIS-SVM model in terms of the operation time will gradually appear with the increase in the number of processing samples in practical production. In terms of Ac, the Ac values of GLCM-based models were greater than $95 \%$, while the Ac values of HIS-based models were between 80 and $90 \%$. It indicated that the GLCM-based models were more advantageous in the identification of different levels of salted sea cucumber, i.e., the image texture features of GLCM were more significantly affected by changes in salt content. Besides, the Se values of GLCM-GRNN and GLCM-SVM models were $100 \%$, indicating that the two models had a strong ability to identify unqualified samples (G2). Similarly, the Sp values indicated that HIS-SVM and GLCM-SVM models had a strong ability to identify qualified samples (G1). In general, the GLCM feature combined with the SVM model (GLCM-SVM) had the best discrimination effect. The excellent performance of the model indicated that the method established in this paper was suitable for the identification of unqualified salted sea cucumber. 


\section{Conclusions}

Identification of unqualified salted sea cucumbers was illustrated based on pressure contact by combining image texture of contour variation with machine learning methods. A method was established to exert standard and uniform pressure contact, and image data of sea cucumber contour variation during the recovery process were collected after pressure removal. An image processing method specifically for the image texture of sea cucumber contour variation was constructed, and the dimensionality reduction and integration of contour image data were achieved. Based on this, HIS and GLCM image texture features integrated with GRNN and SVM were used to establish the identification models (HIS-GRNN, GLCM-GRNN, HIS-SVM, and GLCM-SVM). After comparison, GLCM-SVM had the best detection effect, and its Ac, Se, and Sp were $100 \%, 100 \%$, and $100 \%$, respectively, indicating that this method had a better identification effect for unqualified and qualified salted sea cucumbers. The aforementioned research results proved that the contour variation feature of sea cucumbers during the recovery process combined with a specific machine learning method could achieve the objective, rapid, and nondestructive adulteration detection of salted sea cucumbers. In subsequent studies, the performance of the identification model can be further improved by increasing the number of samples. Further, quantitative prediction of sample texture quality can be achieved by including traditional texture property data.

\section{Data Availability}

The data used to support the findings of this study are available from the corresponding author upon request.

\section{Conflicts of Interest}

The authors declare no conflicts of interest.

\section{Acknowledgments}

This work was supported by the Natural Science Foundation of China (31701696), the Natural Science Foundation of Liaoning Province (2020-MS-273 and 20180551017), and the Innovative Support Program for High-level Personnel of Dalian (2017RQ128).

\section{References}

[1] C. Li, H. Li, S. Guo, X. Li, and X. Zhu, "Evaluation of processing methods on the nutritional quality of sea cucumber (Apostichopus japonicusSelenka)," Journal of Aquatic Food Product Technology, vol. 27, no. 4, pp. 406-417, 2018.

[2] J. Wen, C. Hu, and S. Fan, "Chemical composition and nutritional quality of sea cucumbers," Journal of the Science of Food and Agriculture, vol. 90, no. 14, pp. 2469-2474, 2010.

[3] Z. Liu, Y. X. Liu, D. Y. Zhou et al., "The role of matrix metalloprotease (MMP) to the autolysis of sea cucumber (Stichopus japonicus)," Journal of the Science of Food and Agriculture, vol. 99, no. 13, pp. 5752-5759, 2019.
[4] Y.-X. Liu, D. Y. Zhou, Z. Q. Liu et al., "Structural and biochemical changes in dermis of sea cucumber (Stichopus japonicus) during autolysis in response to cutting the body wall," Food Chemistry, vol. 240, pp. 1254-1261, 2018.

[5] M. Li, Y. Qi, L. Mu et al., "Effects of processing method on chemical compositions and nutritional quality of ready-toeat sea cucumber (Apostichopus japonicus)," Food Science \& Nutrition, vol. 7, no. 2, pp. 755-763, 2019.

[6] B. L. Gianasi, J. F. Hamel, and A. Mercier, "Experimental test of optimal holding conditions for live transport of temperate sea cucumbers," Fisheries Research, vol. 174, pp. 298-308, 2016.

[7] J. H. Moon and W. B. Yoon, "Size dependence of the salting process for dry salted sea cucumber (Stichopus japonicus)," Journal of Food Engineering, vol. 170, pp. 170-178, 2016.

[8] W. Zhu, L. Wang, Y. Guo, X. Li, X. Gu, and L. He, "Analysis of formulation and revision of industrial standards for salted sea cucumber (Stichepus japonicus)," Chinese Fishery Quality and Standards, vol. 5, pp. 31-37, 2015.

[9] M. Cluff, I. A. Kobane, C. Bothma, C. J. Hugo, and A. Hugo, "Intermediate added salt levels as sodium reduction strategy: effects on chemical, microbial, textural and sensory quality of polony," Meat Science, vol. 133, pp. 143-150, 2017.

[10] E. K. McMurtrie, S. D. Johanningsmeier, F. Breidt Jr., and R. E. Price, "Effect of brine acidification on fermentation microbiota, chemistry, and texture quality of cucumbers fermented in calcium or sodium chloride brines," Journal of Food Science, vol. 84, no. 5, pp. 1129-1137, 2019.

[11] O. K. Topuz, "Effects of marinating time, acetic acid and salt concentrations on the quality of little tunny fish (Euthynnus alletteratus) fillet," Journal of Food Processing \& Preservation, vol. 40, no. 6, pp. 1154-1163, 2016.

[12] M. Chabbouh, S. Ben Hadj Ahmed, A. Farhat, A. Sahli, and S. Bellagha, "Studies on the salting step of Tunisian kaddid meat: experimental kinetics, modeling and quality," Food and Bioprocess Technology, vol. 5, no. 5, pp. 1882-1895, 2012.

[13] L. F. Krzeminski, A. Rartal, and W. A. Landmann, "Rapid potentiometric method for determining sodium chloride in cured meat," Journal of Food Science, vol. 30, no. 1, pp. 52-56, 1965.

[14] L. R. B. Mariutti and N. Bragagnolo, "Influence of salt on lipid oxidation in meat and seafood products: a review," Food Research International, vol. 94, pp. 90-100, 2017.

[15] K. A. Thorarinsdottir, S. Arason, S. Sigurgisladottir, T. Valsdottir, and E. Tornberg, "Effects of different pre-salting methods on protein aggregation during heavy salting of cod fillets," Food Chemistry, vol. 124, no. 1, pp. 7-14, 2011.

[16] X. Dong, W. Liu, X. Song et al., "Characterization of heatinduced water adsorption of sea cucumber body wall," Journal of Food Science, vol. 84, no. 1, pp. 92-100, 2019.

[17] J. Wang, L. Lin, X. Sun, and H. Hou, "Mechanism of sea cucumbers (Apostichopus japonicus) body wall changes under different thermal treatment at micro-scale," LWT-Food Science \& Technology, vol. 130, p. 109461, 2020.

[18] S. Geng, H. Wang, X. Wang et al., "A non-invasive NMR and MRI method to analyze the rehydration of dried sea cucumber," Analytical Methods, vol. 7, no. 6, pp. 2413-2419, 2015.

[19] F. Bajd, M. Škrlep, M. Čandek-Potokar, and I. Serša, "MRIaided texture analyses of compressed meat products," Journal of Food Engineering, vol. 207, pp. 108-118, 2017.

[20] Y.-N. Du, X. K. Guo, Y. T. Han et al., "Physicochemical and functional properties of protein isolate from sea cucumber 
(Stichopus japonicus) guts," Journal of Food Processing \& Preservation, vol. 43, no. 7, 2019.

[21] Y. Khotimchenko, "Pharmacological potential of sea cucumbers," International Journal of Molecular Sciences, vol. 19, article 1342, no. 5, 2018.

[22] H. Wang, Y. Zheng, W. Gu et al., "Assessment on the sea cucumber freshness based on the fusion of tactile features and visual textures," Carpathian Journal of Food Science \& Technology, vol. 7, no. 4, pp. 145-153, 2015.

[23] T. Brosnan and D.-W. Sun, "Improving quality inspection of food products by computer vision--a review," Journal of Food Engineering, vol. 61, no. 1, pp. 3-16, 2004.

[24] A. Issac, M. K. Dutta, and B. Sarkar, "Computer vision based method for quality and freshness check for fish from segmented gills," Computers and Electronics in Agriculture, vol. 139, pp. 10-21, 2017.

[25] D. P. Cavallo, M. Cefola, B. Pace, A. F. Logrieco, and G. Attolico, "Non-destructive and contactless quality evaluation of table grapes by a computer vision system," Computers \& Electronics in Agriculture, vol. 156, pp. 558-564, 2019.

[26] X. Zou, J. Zhao, and Y. Li, "Apple color grading based on organization feature parameters," Pattern Recognition Letters, vol. 28, no. 15, pp. 2046-2053, 2007.

[27] I. Paulus, R. de Busscher, and E. Schrevens, "Use of image analysis to investigate human quality classification of apples," Journal of Agricultural Engineering Research, vol. 68, no. 4, pp. 341-353, 1997.

[28] N. Razmjooy, B. S. Mousavi, and F. Soleymani, “A real-time mathematical computer method for potato inspection using machine vision," Computers \& Mathematics with Applications, vol. 63, no. 1, pp. 268-279, 2012.

[29] E. M. Achata, E. S. Inguglia, C. A. Esquerre, B. K. Tiwari, and C. P. O'Donnell, "Evaluation of Vis-NIR hyperspectral imaging as a process analytical tool to classify brined pork samples and predict brining salt concentration," Journal of Food Engineering, vol. 246, pp. 134-140, 2019.

[30] G. Jin, L. He, Q. Wang et al., "Pulsed pressure assisted brining of porcine meat," Innovative Food Science \& Emerging Technologies, vol. 22, pp. 76-80, 2014.

[31] Z. Chen, Z. Changli, S. Weizheng, and C. Xiaoxia, "Grading method of leaf spot disease based on image processing," Journal of Agricultural Mechanization Research, vol. 11, pp. 73-75, 2008.

[32] C. Chen, J. Liang, H. Zhao, H. Hu, and J. Tian, "Frame difference energy image for gait recognition with incomplete silhouettes," Pattern Recognition Letters, vol. 30, no. 11, pp. 977-984, 2009.

[33] J. Han and B. Bhanu, "Individual recognition using gait energy image," IEEE Transactions on Pattern Analysis \& Machine Intelligence, vol. 28, no. 2, pp. 316-322, 2006.

[34] R. M. Haralick, K. Shanmugam, and I. Dinstein, "Textural features for image classification," Studies in Media and Communication, vol. SMC-3, no. 6, pp. 610-621, 1973.

[35] J. Jing, M. Xu, P. Li, Q. Li, and S. Liu, "Automatic classification of woven fabric structure based on texture feature and PNN," Fibers and Polymers, vol. 15, no. 5, pp. 1092-1098, 2014.

[36] H. Zhang, Q. Li, J. Liu et al., "Object-based crop classification using multi-temporal SPOT-5 imagery and textural features with a random forest classifier," Geocarto International, vol. 33, no. 10, pp. 1017-1035, 2017.

[37] L. Armi and S. Fekri-Ershad, Texture image analysis and texture classification methods - a review, 2019, http://arxiv.org/ abs/1904.06554.
[38] F. Tajeri pour, M. Rezaei, M. Saberi, and S. F. Ershad, "Texture classification approach based on combination of random threshold vector technique and co-occurrence matrixes. in Computer Science and Network Technology (ICCSNT)," in Proceedings of 2011 International Conference on Computer Science and Network Technology, Harbin, China, December 2011.

[39] R. C. Gonzalez, R. E. Woods, and S. L. Eddins, Digital Image Processing Using MATLAB, Publishing House of Electronics Industry, 2nd edition, 2009.

[40] O. Holub and S. T. Ferreira, "Quantitative histogram analysis of images," Computer Physics Communications, vol. 175, no. 9, pp. 620-623, 2006.

[41] X. Ou, W. Pan, and P. Xiao, "In vivo skin capacitive imaging analysis by using grey level co-occurrence matrix (GLCM)," International Journal of Pharmaceutics, vol. 460, no. 1-2, pp. 28-32, 2014.

[42] Y. Hu, Z. Wang, X. Fan, J. Li, and A. Gao, "Material microstructures analyzed by using gray level co-occurrence matrices," Chinese Physics B, vol. 26, article 10.1088/1674-1056/26/9/098104, pp. 483-490, 2017.

[43] Y. Park and J. M. Guldmann, "Measuring continuous landscape patterns with gray-level co-occurrence matrix (GLCM) indices: an alternative to patch metrics?," Ecological Indicators, vol. 109, pp. 105802-105818, 2020.

[44] E. O. Olaniyi, A. A. Adekunle, T. Odekuoye, and A. Khashman, "Automatic system for grading banana using GLCM texture feature extraction and neural network arbitrations," Journal of Food Process Engineering, vol. 40, no. 6, pp. 1-10, 2017.

[45] S. Abirami, V. Ramalingam, and S. Palanivel, "Species classification of aquatic plants using GRNN and BPNN," AI \& Society, vol. 29, no. 1, pp. 45-52, 2014.

[46] S. Li, J. T. Kwok, H. Zhu, and Y. Wang, "Texture classification using the support vector machines," Pattern Recognition, vol. 36, no. 12, pp. 2883-2893, 2003.

[47] J. Liu, S. Liu, T. Shi et al., "A modified feature fusion method for distinguishing seed strains using hyperspectral data," International Journal of Food Engineering, vol. 16, no. 7, 2020.

[48] Y. Chen, L. Zheng, J. Huang, Z. Zou, and C. Li, "Prediction of gas emission based on grey-generalized regression neural network," IOP Conference Series: Earth and Environmental Science, vol. 467, article 012056, 2020.

[49] B. Li, M. Liu, Z. Guo, and Y. Ji, "Mechanical fault diagnosis of high voltage circuit breakers utilizing EWT-improved time frequency entropy and optimal GRNN classifier," Entropy, vol. 20 , no. 6 , p. $448,2018$.

[50] J. Liu, M. Zuo, S. S. Low et al., "Fuzzy evaluation output of taste information for liquor using electronic tongue based on cloud model," Sensors, vol. 20, no. 3, p. 686, 2020.

[51] Q. Wang, J. Wang, M. Zhou, Q. Li, and Y. Wang, "Spectralspatial feature-based neural network method for acute lymphoblastic leukemia cell identification via microscopic hyperspectral imaging technology," Biomedical Optics Express, vol. 8, no. 6, pp. 3017-3028, 2017.

[52] J. M. Balage, J. M. Amigo, D. S. Antonelo, M. R. Mazon, and S. da Luz e Silva, "Shear force analysis by core location in Longissimus steaks from Nellore cattle using hyperspectral images - a feasibility study," Meat Science, vol. 143, pp. 30-38, 2018. 
[53] T. Y. Goh, S. N. Basah, H. Yazid, M. J. Aziz Safar, and F. S. Ahmad Saad, "Performance analysis of image thresholding: Otsu technique," Measurement, vol. 114, pp. 298-307, 2018.

[54] N. Guizani, A. O. al-Shoukri, A. Mothershaw, and M. S. Rahman, "Effects of salting and drying on shark (Carcharhinus sorrah) meat quality characteristics," Drying Technology, vol. 26, no. 6, pp. 705-713, 2008. 